\title{
Low-pressure gas breakdown in uniform dc electric field
}

\author{
V A Lisovskiy, S D Yakovin and V D Yegorenkov \\ Kharkov National University, 4, Svobody Square, Kharkov, 61077, Ukraine
}

Received 7 April 2000, in final form 28 July 2000

\begin{abstract}
This paper studies in experiment and theory the breakdown of argon, nitrogen, air and oxygen in a uniform dc electric field at different discharge gaps $L$, discharge tube radii $R$ and cathode materials. At arbitrary geometric dimensions of the cylindrical discharge vessel and cathode materials the ratio of the breakdown electric field value to the gas pressure $p$ at the minimum of the breakdown curves is shown to remain constant, $\left(E_{d c} / p\right)_{\min } \approx$ constant. A modified breakdown law for the low-pressure dc discharge $U_{d c}=f(p L, L / R)$ is obtained. That is, the breakdown voltage $U_{d c}$ is shown to depend not only on the product $p L$, but also on the ratio $L / R$. A method is presented enabling one to predict a dc breakdown curve in the cylindrical discharge vessel possessing arbitrary $L$ and $R$ values from the measured data on the dc breakdown.
\end{abstract}

\section{Introduction}

Dc glow discharges are widely applied for depositing thin polymer and oxide films, for cleaning the surface of materials, for pumping gas discharge lasers, in plasma display panels, voltage stabilizers, etc. Therefore the research into the conditions of the glow discharge breakdown is of considerable interest.

Ignition of the dc glow discharge is one of the oldest problems in the study of low-pressure gas discharges. As far as it is known [1-12], the breakdown curves of the glow discharge are described by Paschen's law $U_{d c}=f(p L)$, i.e. the breakdown voltage $U_{d c}$ depends on the product of the gas pressure $p$ and the discharge gap $L$. De La Rue and Muller [13] were the first to observe the dependence of the breakdown voltage $U_{d c}$ on the product $p L$. Later Paschen measured the breakdown curves of the dc discharge in air, $\mathrm{CO}_{2}$ and $\mathrm{H}_{2}$ over a broad range of $p L$ values and came to the conclusion that the breakdown voltage $U_{d c}$ depends only on the product $p L$ [1]. Paschen's law means that the breakdown curves $U_{d c}(p)$, measured for various gaps $L$, should superimpose onto each other, if one depicts them as $U_{d c}(p L)$. However, Townsend and McCallum [14] measured the breakdown curves of the dc discharge in neon and discovered that for equal values of the product $p L$ the breakdown voltage associated with a larger discharge gap with planar electrodes is considerably higher than that associated with a smaller gap. Later studies [15-23] confirmed this conclusion for a number of gases (neon, argon, nitrogen, hydrogen and others). Despite the large number of experimental and theoretical papers devoted to the lowpressure gas breakdown in the dc electric field, there has been until now no method capable of predicting the breakdown curve for arbitrary discharge gaps $L$ and discharge vessel radii $R$ from measured data.
This paper reports the results of experimental and theoretical study of breaking down argon, nitrogen, air and oxygen in the dc electric field in cylindrical discharge vessels with various interelectrode gaps $L$, inner radii $R$ and cathode materials. On increasing the discharge gap $L$ or decreasing the radius of the discharge tube $R$ the breakdown curves are shown to shift to the region of higher $p L$ values and higher breakdown voltage values $U_{d c}$. Still, the ratio of the breakdown electric field value to the gas pressure value at the breakdown curve minimum $\left(E_{d c} / p\right)_{\min }$ remains constant at any value of the discharge gap $L$, discharge tube radius $R$ and the ion-electron emission rate $\gamma$. We have obtained a modified breakdown law for the dc low-pressure discharge: $U_{d c}=f(p L, L / R)$. A method is described for the determination of the breakdown curve of a de discharge in a cylindrical vessel with arbitrary geometric dimensions from the breakdown curve for a narrow discharge gap (at $L / R \rightarrow 0$ ), i.e. from the conventional Paschen's curve.

\section{Theoretical treatment}

For the theoretical description of the dc gas breakdown the Townsend equation [2] is presently widely used:

$$
\gamma[\exp (\alpha L)-1]=1
$$

where $\alpha$ and $\gamma$ are the first and the second Townsend coefficients. This simple equation was derived for planar electrodes with an infinitely large radius $R$, i.e. for $L / R=0$. However, in actual gas discharge devices (both research or technology relevant) the radius of the electrodes may be comparable to the interelectrode gap. Specifically, such a situation is found in some designs of the plasma display panels [24-29]. Therefore, for the description of the dc discharge breakdown in such devices, one should obtain the gas breakdown criterion, taking into account the escape of 
charged particles to the lateral walls of the discharge tube (to the cells in the plasma display panel, etc). The equation for the gas breakdown in a dc uniform electric field obtained in [30] takes into account the ionization of gas molecules through electron impact and electron and ion drift along the field as well as the diffusional escape of electrons along the radius of the discharge tube. However, the authors of [30] performed neither an analysis of the breakdown equation obtained nor a comparison of the calculations with measured data and, even, gave no theoretical breakdown curve. Therefore, having made a simple transformation, we present the equation (12) of [30] in the form:

$$
\alpha=\frac{D_{e}}{V_{e}}\left(\frac{2.4}{R}\right)^{2}+\alpha \gamma\left\{\exp \left[L\left(\alpha-\frac{D_{e}}{V_{e}}\left(\frac{2.4}{R}\right)^{2}\right)\right]-1\right\}
$$

where $D_{e}$ is the coefficient of the transverse diffusion of electrons, $V_{e}$ is the electron-drift velocity. We will use the conventional notation for $V_{e}, D_{e}$ and $\alpha$ :

$$
\begin{gathered}
V_{e}=\mu_{e} E_{d c}=\mu_{e 0} \frac{U_{d c}}{p L} \\
D_{e}=D_{e 0} / p \\
\alpha=A_{0} p \exp \left(-\frac{B_{0} p L}{U_{d c}}\right)
\end{gathered}
$$

where $\mu_{e 0}$ and $D_{e 0}$ are the mobility and the coefficient of the transverse diffusion of electrons at $p=1$ Torr, respectively, and $A_{0}$ and $B_{0}$ are constants [5]. Let us insert the expressions (3)-(5) into (2) and multiply the left- and right-hand parts of (2) by $L$; then we obtain the following equation for the breakdown:

$$
\begin{aligned}
& A_{0}(p L) \exp \left(-\frac{B_{0}(p L)}{U_{d c}}\right)=\frac{D_{e 0}}{\mu_{e 0}} \frac{(2.4)^{2}}{U_{d c}}\left(\frac{L}{R}\right)^{2} \\
& +\gamma A_{0}(p L) \exp \left(-\frac{B_{0}(p L)}{U_{d c}}\right) \\
& \quad \times\left\{\operatorname { e x p } \left[A_{0}(p L) \exp \left(-\frac{B_{0}(p L)}{U_{d c}}\right)\right.\right. \\
& \left.\left.-\frac{D_{e 0}}{\mu_{e 0}} \frac{(2.4)^{2}}{U_{d c}}\left(\frac{L}{R}\right)^{2}\right]-1\right\} .
\end{aligned}
$$

One sees from (6) that the breakdown voltage $U_{d c}$ depends not only on the product $p L$, but also on the ratio $L / R$.

Let us differentiate expression (6) over $p L$ and make the derivative $\mathrm{d} U_{d c} / \mathrm{d}(p L)$ equal to zero. Then, for the minimum of the breakdown curve we obtain two solutions, one of which makes no sense physically and another that gives the following relations:

$$
\begin{aligned}
& \left(\frac{U_{d c}}{p L}\right)_{\text {min }}=\left(\frac{E_{d c}}{p}\right)_{\text {min }}=B_{0} \\
& \frac{A_{0}}{\mathrm{e}}(p L)_{\min }=\frac{D_{e 0}}{\mu_{e 0}} \frac{(2.4)^{2}}{B_{0}(p L)_{\min }}\left(\frac{L}{R}\right)^{2}+\frac{\gamma A_{0}}{\mathrm{e}}(p L)_{\min } \\
& \quad \times\left\{\exp \left[\frac{A_{0}}{\mathrm{e}}(p L)_{\min }-\frac{D_{e 0}}{\mu_{e 0}} \frac{(2.4)^{2}}{B_{0}(p L)_{\min }}\left(\frac{L}{R}\right)^{2}\right]-1\right\}
\end{aligned}
$$

$$
\frac{A_{0}}{\mathrm{e} B_{0}} U_{\min }=\frac{D_{e 0}}{\mu_{e 0}} \frac{(2.4)^{2}}{U_{\min }}\left(\frac{L}{R}\right)^{2}+\frac{\gamma A_{0}}{\mathrm{e}} \frac{U_{\min }}{B_{0}}
$$

$$
\times\left\{\exp \left[\frac{A_{0}}{\mathrm{e} B_{0}} U_{\text {min }}-\frac{D_{e 0}}{\mu_{e 0}} \frac{(2.4)^{2}}{U_{\min }}\left(\frac{L}{R}\right)^{2}\right]-1\right\}
$$

where e is the base of natural logarithms, $U_{\min }=\left(U_{d c}\right)_{\min }$. At $L / R \rightarrow 0$, expressions (8) and (9) assume the form [5]:

$$
\begin{gathered}
(p L)_{\text {min }}=\frac{\mathrm{e}}{A_{0}} \ln \left(\frac{1+\gamma}{\gamma}\right) \\
U_{\text {min }}=\frac{\mathrm{e} B_{0}}{A_{0}} \ln \left(\frac{1+\gamma}{\gamma}\right) .
\end{gathered}
$$

Note that expressions (7)-(9) were obtained for the case when the ratio $D_{e 0} / \mu_{e 0}$ does not depend on the ratio $E_{d c} / p$. This is valid for heavy noble gases-argon, xenon-in a broad range of $E_{d c} / p$ values corresponding to the section near to and to the left of the breakdown curve of a dc discharge. However, it is easy to prove that taking the relation $D_{e 0} / \mu_{e 0}=f\left(E_{d c} / p\right)$ into account leads eventually to the expressions (7)-(9), indicating their universal nature. Moreover, expression (7) also remains valid in the case when one takes into account the anisotropic diffusion of electrons along and across the direction of the electric field and the photoemission of electrons from the cathode surface.

Note that expression (5) provides a satisfactory description of the first Townsend coefficient on the ratio of the dc electric field strength to the gas pressure $\alpha\left(E_{d c} / p\right)$ for molecular gases. For noble gases one often uses the following dependence $[12,31]$ :

$$
\alpha=D p \exp \left[-C \cdot\left(E_{d c} / p\right)^{-1 / 2}\right]
$$

where $D$ and $C$ are the constants related to the gas species. Inserting expression (12) into expression (2) yields the following relations at the breakdown curve minimum:

$$
\begin{aligned}
& \left(\frac{U_{d c}}{p L}\right)_{\text {min }}=\left(\frac{E_{d c}}{p}\right)_{\text {min }}=\frac{C^{2}}{4}=\text { constant } \\
& \frac{D}{\mathrm{e}^{2}}(p L)_{\min }=\frac{D_{e 0}}{\mu_{e 0}} \frac{4 \cdot(2.4)^{2}}{C^{2}(p L)_{\min }}\left(\frac{L}{R}\right)^{2}+\frac{\gamma D}{\mathrm{e}^{2}}(p L)_{\min } \\
& \quad \times\left\{\exp \left[\frac{D}{\mathrm{e}^{2}}(p L)_{\min }-\frac{D_{e 0}}{\mu_{e 0}} \frac{4(2.4)^{2}}{C^{2}(p L)_{\min }}\left(\frac{L}{R}\right)^{2}\right]-1\right\} \\
& \frac{4 D}{\mathrm{e}^{2} C^{2}} U_{\text {min }}=\frac{D_{e 0}}{\mu_{e 0}} \frac{(2.4)^{2}}{U_{\text {min }}}\left(\frac{L}{R}\right)^{2}+\gamma \frac{4 D}{\mathrm{e}^{2} C^{2}} U_{\min } \\
& \quad \times\left\{\exp \left[\frac{4 D}{\mathrm{e}^{2} C^{2}} U_{\text {min }}-\frac{D_{e 0}}{\mu_{e 0}} \frac{(2.4)^{2}}{U_{\text {min }}}\left(\frac{L}{R}\right)^{2}\right]-1\right\}
\end{aligned}
$$

Therefore, it follows from expressions (7) and (13) that on changing the values of $L, R$ and $\gamma$ of the discharge vessel, the breakdown curves are shifted in such a way that the quantity $\left(E_{d c} / p\right)_{\min }$ always remains constant at the minimum of them, the ionization ability of electrons being a maximum. It is seen from (8), (9), (14) and (15) that the coordinates of the minimum $(p L)_{\min }$ and $U_{\min }$ depend on the ratio $L / R$ and not on $L$ and $R$ separately.

\section{Experimental details}

We measured the breakdown curves of the glow discharge in argon, nitrogen, air and oxygen within the range of dc 


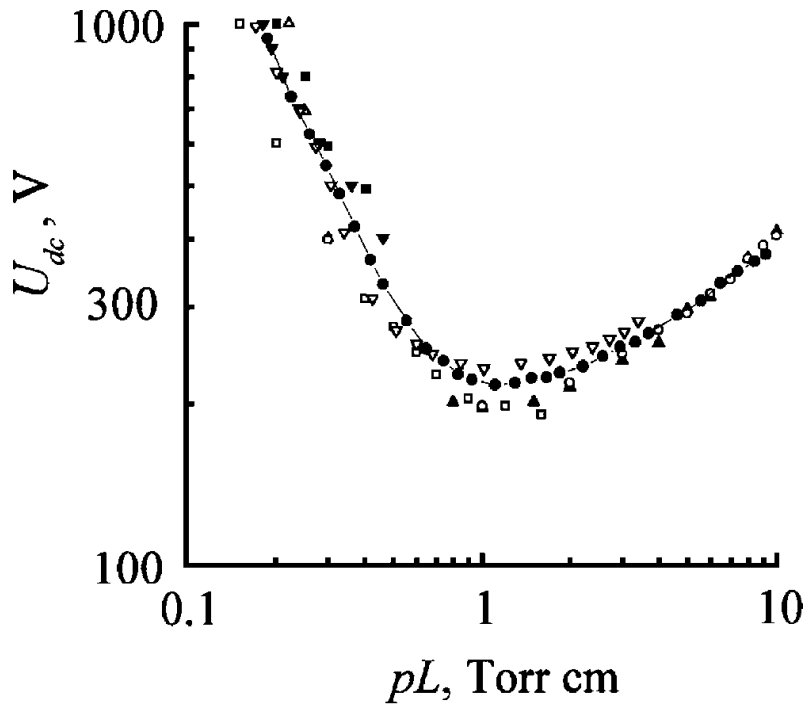

Figure 1. Measured breakdown curves of the glow discharge in argon: $\bullet$ presents our data $(L=1.1 \mathrm{~cm}, R=5 \mathrm{~cm}$, stainless-steel cathode; $\bigcirc$ data from the experiment of [36] (platinum cathode): $\Delta$ is taken from [32] (nickel cathode); $\Delta$ is from [33] (steel cathode); $\boldsymbol{\square}$ is from [34] (nickel cathode); $\square$ is from [35] (steel cathode); $\boldsymbol{\nabla}$ from [37] (platinum cathode); and $\nabla$ is from [19] (copper cathode).

voltage $U_{d c} \leqslant 1000 \mathrm{~V}$ and pressure $p \approx 10^{-2}-10$ Torr. We used discharge tubes with inner diameters of 9, 14, 27, 63 and $100 \mathrm{~mm}$. In all cases the walls of our discharge tubes were insulating (glass or fused silica). The planar parallel electrodes occupied the whole cross section of the discharge tube. The anode was made out of stainless-steel. We employed cathodes made out of stainless-steel, duralumin, silver, magnesium, zinc and iron as well as the iron cathode covered with soot. Before taking measurements we purified the cathode surface by igniting the dc glow discharge in argon at a pressure $p=0.5$ Torr and a discharge current $I_{d c}=20-50 \mathrm{~mA}$ (depending on the diameter of the discharge tube) for $10 \mathrm{~min}$. Under these conditions the ion flux on the cathode is sufficiently large enough to remove the monolayers of gases remaining on the cathode surface after mechanical machining and polishing, but the discharge current value is not yet sufficient to produce the cathode spots that erode the cathode surface.

We employed no external ionization sources and studied exclusively the ignition of the self-sustained dc discharge.

The accuracy of measuring the dc breakdown voltage was $\pm 2 \mathrm{~V}$.

In all cases we employed the following technique of measuring the breakdown curves. We fixed a certain distance between the electrodes $L$ and then measured the breakdown voltage $U_{d c}$ at different gas pressures $p$. We will explain below why this method of measuring the breakdown curves of the dc discharge is correct.

\section{Experimental results}

We will show first that the breakdown curves of the dc discharge we measured are in reasonable agreement with those of other authors. Figure 1 presents one of our

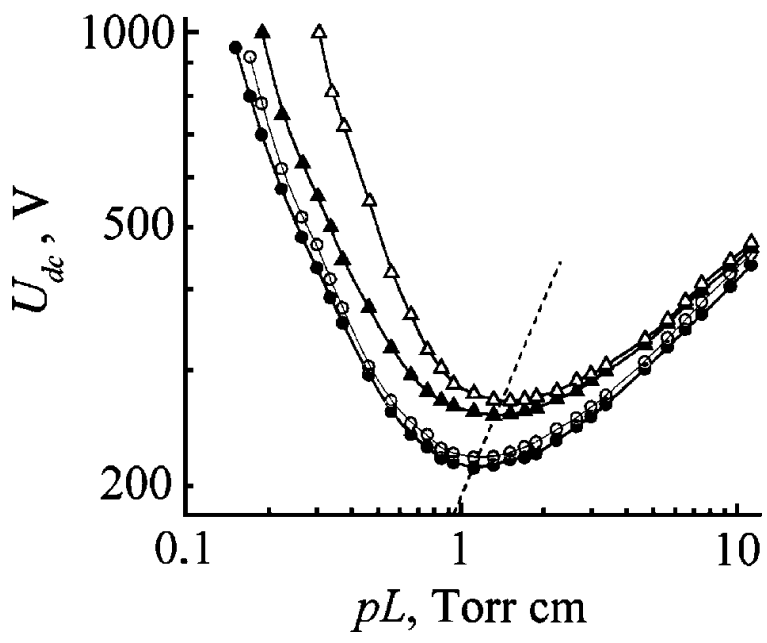

Figure 2. Measured breakdown curves of the glow discharge in argon at $L=0.9 \mathrm{~cm}$ and $R=3.15 \mathrm{~cm}$ : $\bullet$ is for the stainless-steel cathode, $\bigcirc$ is for the silver cathode, $\boldsymbol{\Delta}$ is for the copper cathode and $\Delta$ is for the iron cathode covered with soot.

breakdown curves $(L=11 \mathrm{~mm}$ and $R=50 \mathrm{~mm}$ ) of the dc discharge in argon for the stainless-steel cathode as well as the breakdown curves measured by the authors of [19,3237] with cathodes made out of different materials. This figure manifests the satisfactory agreement between our data and those of other researchers.

Figure 2 shows the breakdown curves of the de discharge we measured in argon with the cathodes made out of different materials. It follows from this figure that for the cathodes with lower ion-electron emisssion rates $\gamma$ (copper, soot) the breakdown curve is shifted simultaneously to higher $p L$ values as well as to higher breakdown voltages $U_{d c}$. Increasing the emission rate of the cathode surface leads to a shift of the breakdown curve to lower $p L$ values as well as lower breakdown voltages $U_{d c}$. At the same time the minima of the breakdown curves we measured are on one straight line. This agrees with the data of $[3,8]$ and also follows from the results of papers $[38,39]$.

Figures 3 and 4 show the breakdown curves for argon and nitrogen we measured with discharge tubes of fixed radius $R$ and different interelectrode gaps $L$. It follows from these figures that on increasing the gap $L$ the curves are shifted not only to the region of higher breakdown voltages $U_{d c}$ (as was established in [14-23]), but simultaneously to higher $p L$ values. A similar conclusion may be drawn from the measured data presented in figure 3 of [19] for neon. The author of [19] measured the breakdown curves near and to the left of the minimum and noted that increasing $L$ leads to the increase of $U_{d c}$. However one can see from the results in [19] that on increasing $L$ the breakdown curves are also shifted to higher $p L$ values (the authors of [19] paid no attention to this fact). Consequently, the deviation from Paschen's law that we have observed is supported by the measured data of other authors. In all probability, this shift of the breakdown curves to higher $U_{d c}$ and $p L$ values with the increase of the interelectrode gap $L$ is associated with the growth of the losses of charged particles on the lateral walls of the discharge tube due to the diffusion across the electric field. 


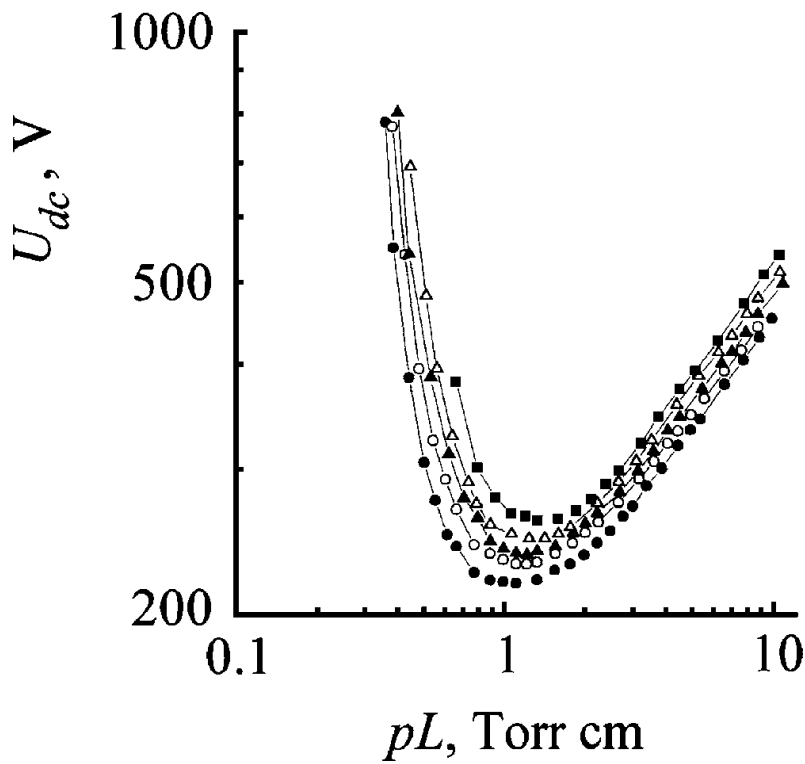

Figure 3. Measured breakdown curves of the glow discharge in $\operatorname{argon}(R=3.15 \mathrm{~cm})$ for the stainless-steel cathode and different interelectrode gaps: $\bullet L=0.5 \mathrm{~cm}, \bigcirc L=1 \mathrm{~cm}, \Delta L=2 \mathrm{~cm}$, $\triangle L=4 \mathrm{~cm}$ and $\mathbf{\square} L=6 \mathrm{~cm}$.

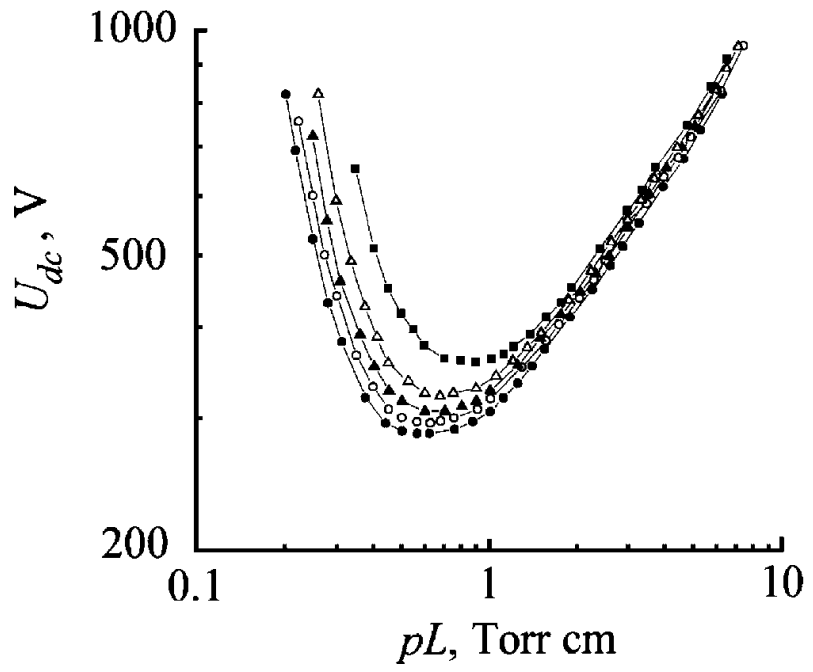

Figure 4. Measured breakdown curves of the glow discharge in nitrogen $(R=3.15 \mathrm{~cm})$ for the stainless-steel cathode and different interelectrode gaps: $\bullet L=0.5 \mathrm{~cm}, \bigcirc L=2 \mathrm{~cm}$, $\Delta L=4 \mathrm{~cm}, \triangle L=6 \mathrm{~cm}$ and $\mathbf{\square} L=8 \mathrm{~cm}$.

Figures 5 and 6 show the dependences of $U_{\min }$ and $\left(E_{d c} / p\right)_{\min }$ on the variables $(p L)_{\min }$ and $L / R$, respectively, obtained from our measured breakdown curves of the dc discharge in argon for different interelectrode gaps $L$ and inner tube radii $R$. In figure 5 one sees that for the measured coordinates of the breakdown curve minima we have $U_{\text {min }} \propto(p L)_{\text {min }}$, and from figures 5 and 6 it follows that $\left(E_{d c} / p\right)_{\text {min }} \approx$ constant $=194 \pm 5 \mathrm{~V}(\mathrm{~cm} \text { Torr })^{-1}$ (this $\left(E_{d c} / p\right)_{\min }$ value is in satisfactory agreement with the value $B_{0}=180 \mathrm{~V}$ (cm Torr $)^{-1}$ [5]). Figure 5 also shows the straight line $U_{\min }=194(p L)_{\min }$, which gives a good description of the measured points. Figure 7 presents the dependences of $U_{\text {min }}$ and $\left(E_{d c} / p\right)_{\text {min }}$ on $(p L)_{\text {min }}$ in nitrogen for different cathode materials and interelectrode gaps $L$.

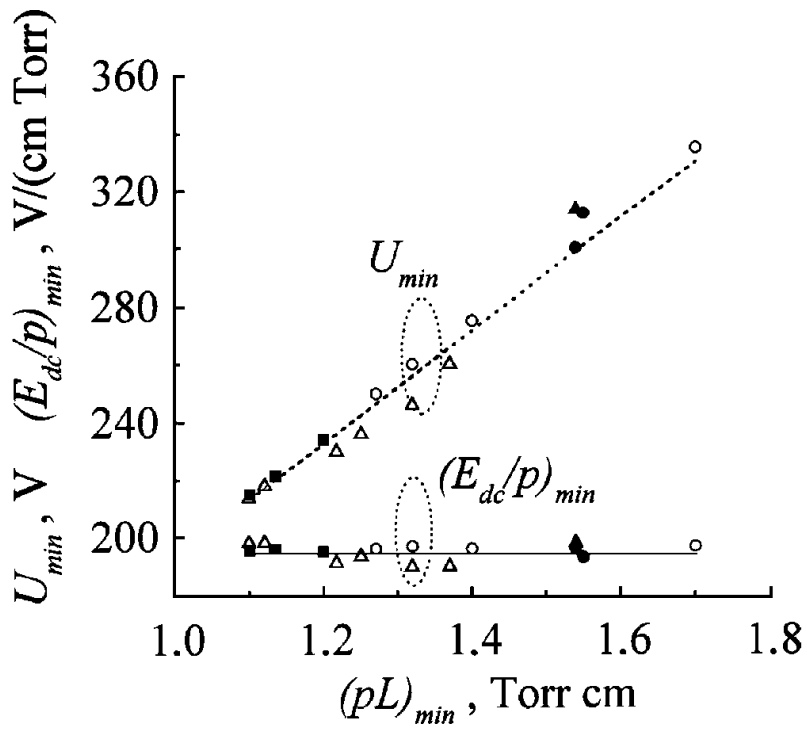

Figure 5. Dependences of $U_{\min }$ and $\left(E_{d c} / p\right)_{\min }$ on $(p L)_{\min }$ in argon for the stainless-steel cathode in discharge tubes with different inner radii: $0.45 \mathrm{~cm}, \bigcirc 0.7 \mathrm{~cm}, \Delta 1.35 \mathrm{~cm}, \triangle 3.15 \mathrm{~cm}$ and $5 \mathrm{~cm}$. The full line is for $\left(E_{d c} / p\right)_{\min }=194 \mathrm{~V}(\mathrm{~cm} \text { Torr })^{-1}$ and the broken line is for $U_{\min }=194(p L)_{\min }$.

The full line in this figure, $U_{\min }=407(p L)_{\min }$, and the broken line, $\left(E_{d c} / p\right)_{\text {min }}=407 \pm 5 \mathrm{~V}$ (cm Torr $)^{-1}$, give a satisfactory description of the results that we have obtained. Therefore the behaviour of the coordinates of the minimum point of the glow discharge breakdown curves predicted by the expression (7) is supported by our measured data. Consequently, for any interelectrode gap $L$ and tube radius $R$ the ratio $\left(E_{d c} / p\right)_{\min }$ at the minimum always remains constant. The same rule holds if one varies the value of the ion-electron secondary emission rate $\gamma$ of the cathode material (this follows from figure 2 and is noted in [3, 4, 8]).

From figure 6 one also sees that the values of $U_{\text {min }}$ obtained from the measured breakdown curves for different $L$ and $R$ values fit satisfactorily one uniformly growing curve. The same figure shows the theoretical curve $U_{\min }(L / R)$, calculated from equation (15), which is in reasonable agreement with our measured data (we used the values of $\alpha$, $V_{e}$ and $D_{e}$ from $\left.[4,5,40-43]\right)$. From figure 6 the conclusion may be drawn that the parameter $L / R$ along with $p L$ is also important in describing the breakdown curves of the glow discharge. Consequently, the gas breakdown law in the dc electric field may be written in the following form:

$$
U_{d c}=f\left(p L^{*}, \frac{L}{R}\right)
$$

where $L^{*}$ is the function of the ratio $L / R$.

Let us now show that the conventional Paschen's law $U_{d c}=f(p L)$ is valid only in two cases: (i) for discharge tubes with $L / R \rightarrow 0$ (this is well known and no proof is required-see [2-9]) and (ii) for discharge tubes with similar geometries $L / R=$ constant (what was predicted by Townsend [2]). Figure 8 presents two breakdown curves that we measured in discharge vessels with different radii. The interelectrode gap was chosen such that the ratio of the interelectrode gap to the tube radius remained constant 


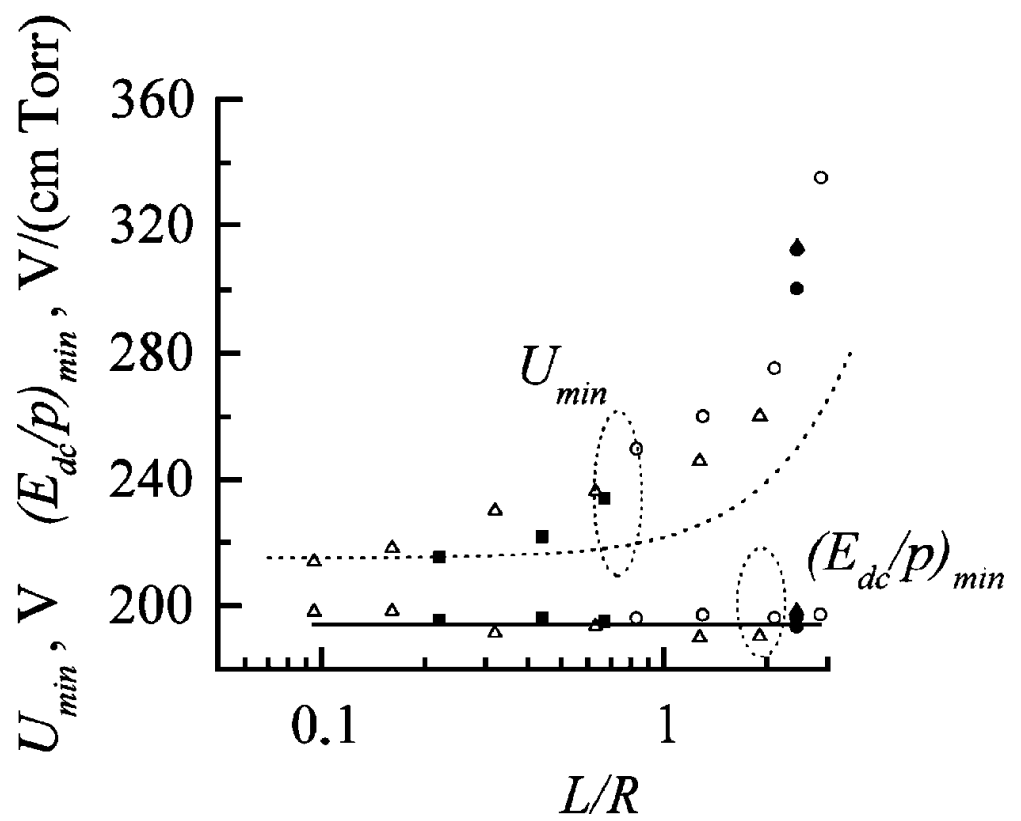

Figure 6. Dependences of $U_{\min }$ and $\left(E_{d c} / p\right)_{\min }$ on $L / R$ in argon in discharge tubes with different inner radii: $\bullet 0.45 \mathrm{~cm}, \bigcirc 0.7 \mathrm{~cm}$, $\Delta 1.35 \mathrm{~cm}, \triangle 3.15 \mathrm{~cm}$ and $\boldsymbol{\square} \mathrm{cm}$. The full curve is for $\left(E_{d c} / p\right)_{\min }=194 \mathrm{~V}(\mathrm{~cm} \text { Torr })^{-1}$ and the broken curve presents the calculation according to (15).

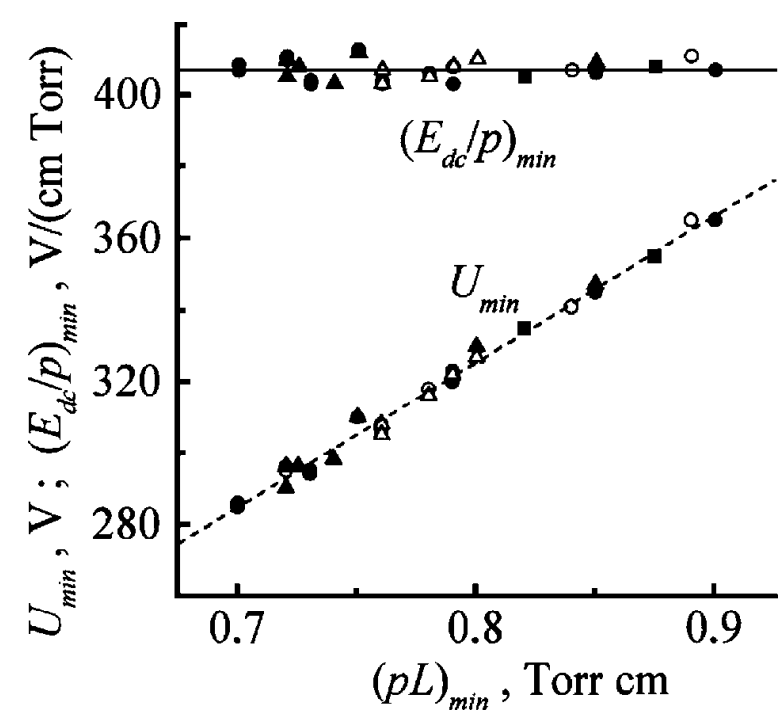

Figure 7. Dependences of $U_{\min }$ and $\left(E_{d c} / p\right)_{\min }$ on $(p L)_{\min }$ in nitrogen in discharge tubes with the inner radius $R=3.15 \mathrm{~cm}$ for different cathodic materials: $\bullet$ is for the stainless-steel cathode, $O$ is for the silver cathode, $\boldsymbol{\Delta}$ is for the aluminium cathode, $\triangle$ is for the magnesium cathode and $\boldsymbol{\square}$ is for the iron cathode covered with soot. The full line presents the dependence $\left(E_{d c} / p\right)_{\min }=407 \mathrm{~V}(\mathrm{~cm} \text { Torr })^{-1}$ and the broken curve is for $U_{\min }=407(p L)_{\min }$.

$L / R=2.4$. It is seen from the figure that in this case the breakdown curves actually superimpose onto each other. The discharges with geometrically similar electrode dimensions and interelectrode gaps possess the same breakdown voltage.

The generalized law, (16) has the following meaning. Take two discharge tubes with $L_{1}, R_{1}$ and $L_{2}, R_{2}$ respectively, measure the breakdown curves for them and draw the curves in the form of the functions $U_{d c 1,2}=f\left(p L_{1,2}\right)$. Then these two curves coincide only when $L_{1} / R_{1}=L_{2} / R_{2}$. In the gen-

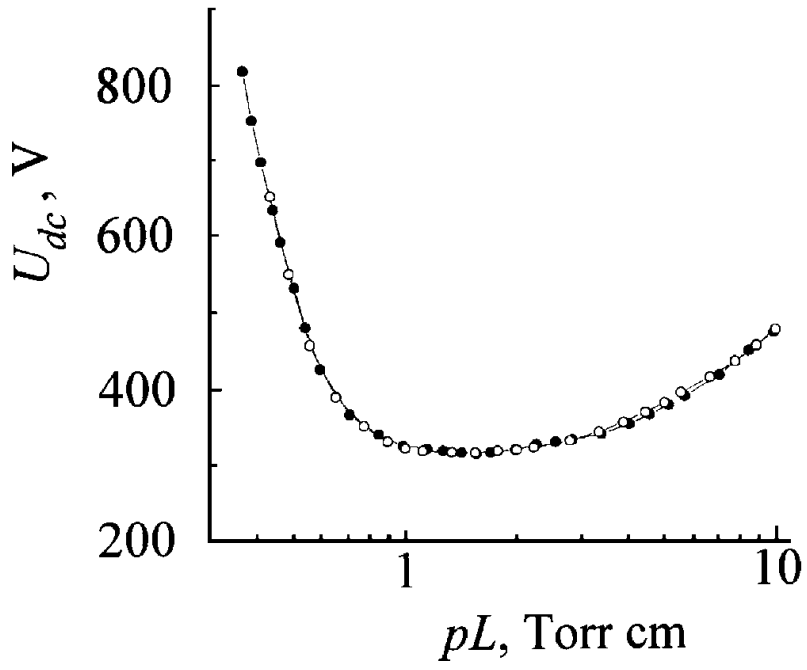

Figure 8. Breakdown curves in argon for the stainless-steel cathode at $L / R=2.4: \bullet L=1.1 \mathrm{~cm}$ and $R=0.45 \mathrm{~cm}$; and $\bigcirc L=3.3 \mathrm{~cm}$ and $R=1.35 \mathrm{~cm}$.

eral case for arbitrary $L$ and $R$ the conventional Paschen's law is not valid and one should apply a more general law of the dc breakdown: (16), which is the short form of the equation (6).

Note that with an appropriate choice of the coordinate axes one can achieve a practical coincidence of all the breakdown curves we have measured (for the same gas and cathode material). For example, if the abscissa is

$$
(p L)^{*}=p L /\left(1+\left(\frac{L}{R}\right)^{2}\right)^{a}
$$

and the ordinate is

$$
U_{d c}^{*}=U_{d c} /\left(1+\left(\frac{L}{R}\right)^{2}\right)^{a}
$$




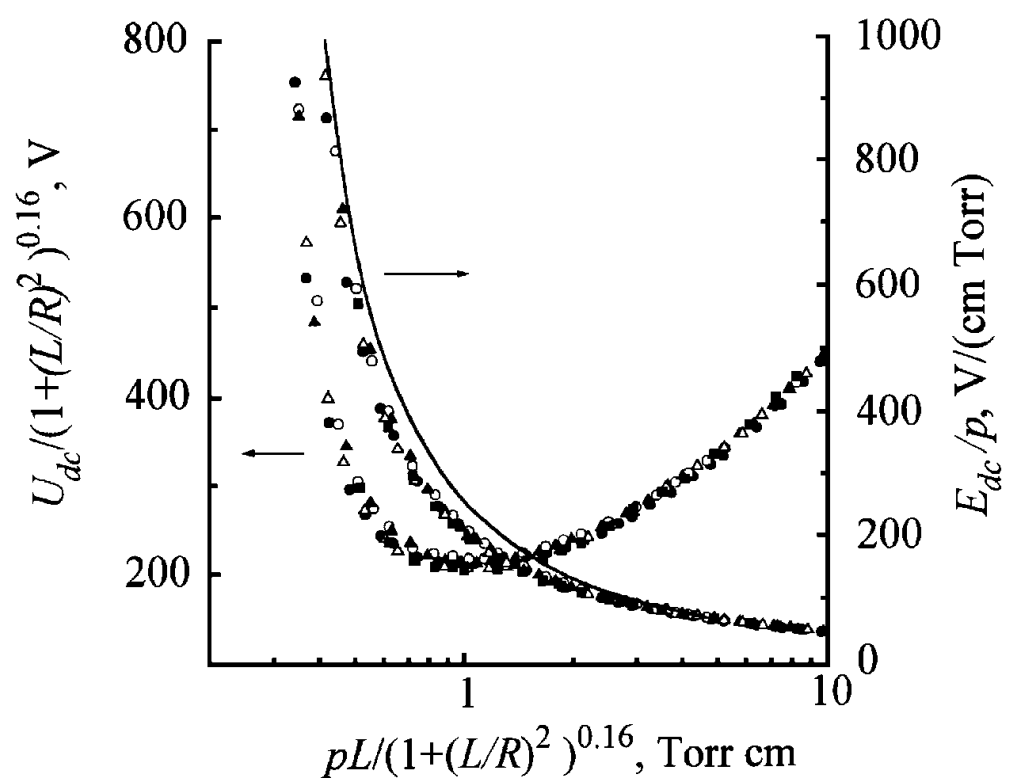

Figure 9. Dependences of $U_{d c}^{*}$ and $E_{d c} / p$ on $(p L)^{*}$ in argon in the discharge tube with $R=3.15 \mathrm{~cm}$ and different interelectrode gaps: $\begin{array}{r}- \\ L\end{array}=0.5 \mathrm{~cm}, \bigcirc L=1 \mathrm{~cm}, \boldsymbol{\Delta} L=2 \mathrm{~cm}, \triangle L=4 \mathrm{~cm}$ and $\boldsymbol{\square} L=6 \mathrm{~cm}$. The full curve presents the calculated data from [44].

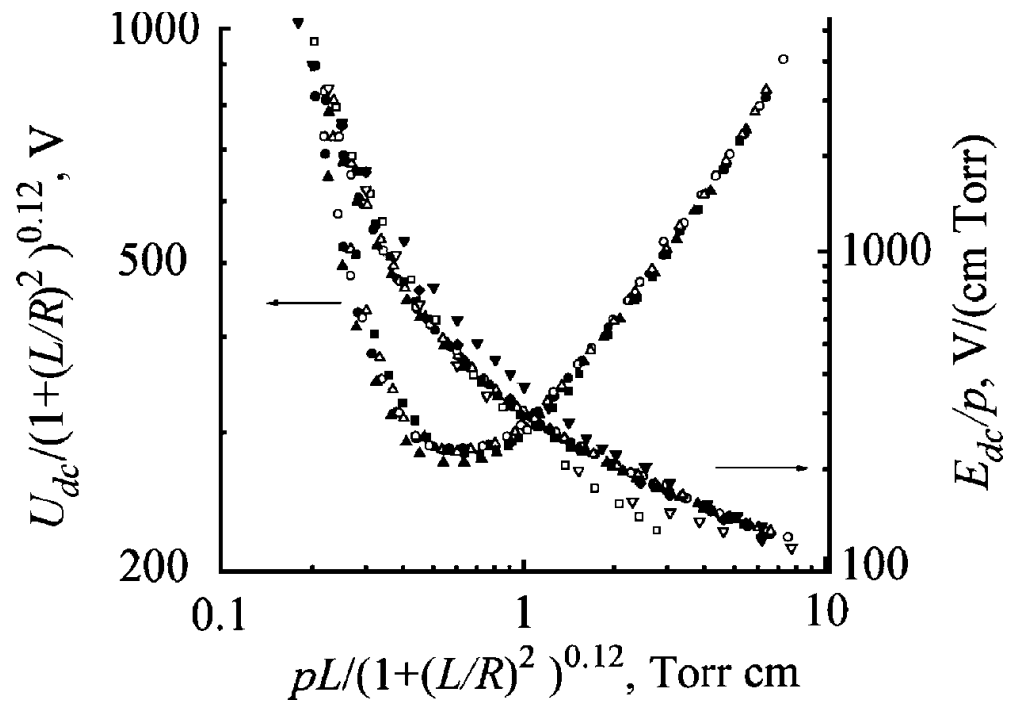

Figure 10. Dependences of $U_{d c}^{*}$ and $E_{d c} / p$ on $(p L)^{*}$ in nitrogen in the discharge tube with $R=3.15 \mathrm{~cm}$ and different interelectrode gaps:

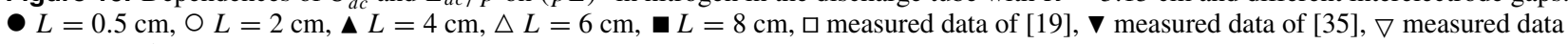
of [45], and $\diamond$ measured data of [46, 47].

where for argon $a \approx 0.16$, then the breakdown curves given in figure 3 coincide to the accuracy of $\pm 5 \mathrm{~V}$ (figure 9). Obviously, for $L / R \rightarrow 0$ we have the conventional Paschen's curve $U_{d c}=f(p L)$. It is seen from (17) and (18), that $U_{d c}^{*} /(p L)^{*}=U_{d c} /(p L)=E_{d c} / p$, i.e. the dependences $E_{d c} / p=f\left((p L)^{*}\right)$ for different breakdown curves should also coincide (as we see in figure 9). Figure 9 also gives the theoretical curve $E_{d c} / p=f\left((p L)^{*}\right)$, obtained in [44] for the case $L / R \rightarrow 0$, which is in reasonable agreement with our data. Figure 10 shows similar dependences for $U_{d c}^{*}=f\left((p L)^{*}\right)$ and $E_{d c} / p=f\left((p L)^{*}\right)$ for nitrogen, the coincidence of the breakdown curves given in figure 4 being observed at $a \approx 0.12$. Here we also witness the reasonable agreement between our data and those of [19,35, 45-47]. For air we have $a \approx 0.09$ (figure 11) and for oxygen one finds $a \approx 0.03$ (figure 12). We obtained the dependences for $E_{d c} / p=f\left((p L)^{*}\right)$ given in figure 11 and 12 from the measured data of $[15,35,45,48,49]$ and they are in satisfactory agreement with our data. For neon we obtained $a \approx 0.11$ from the measured data of [19]. From figures 9-12 it follows that the gas dc breakdown law may also be written in the form $U_{d c}^{*}=f\left((p L)^{*}\right)$.

With the help of the relationships (17) and (18) and the values of the breakdown voltages given in figures 9-12 we can predict with good accuracy the breakdown curves in any cylindrical discharge vessel for any interelectrode gap $L$ and tube radius $R$. For example, let us determine the location of the breakdown curve minimum of the de discharge in nitrogen at $R=3.15 \mathrm{~cm}$ and $L=5 \mathrm{~cm}$ for the stainlesssteel cathode. It is seen from figure 10 that $U_{\text {min }}^{*} \approx 280 \mathrm{~V}$, 


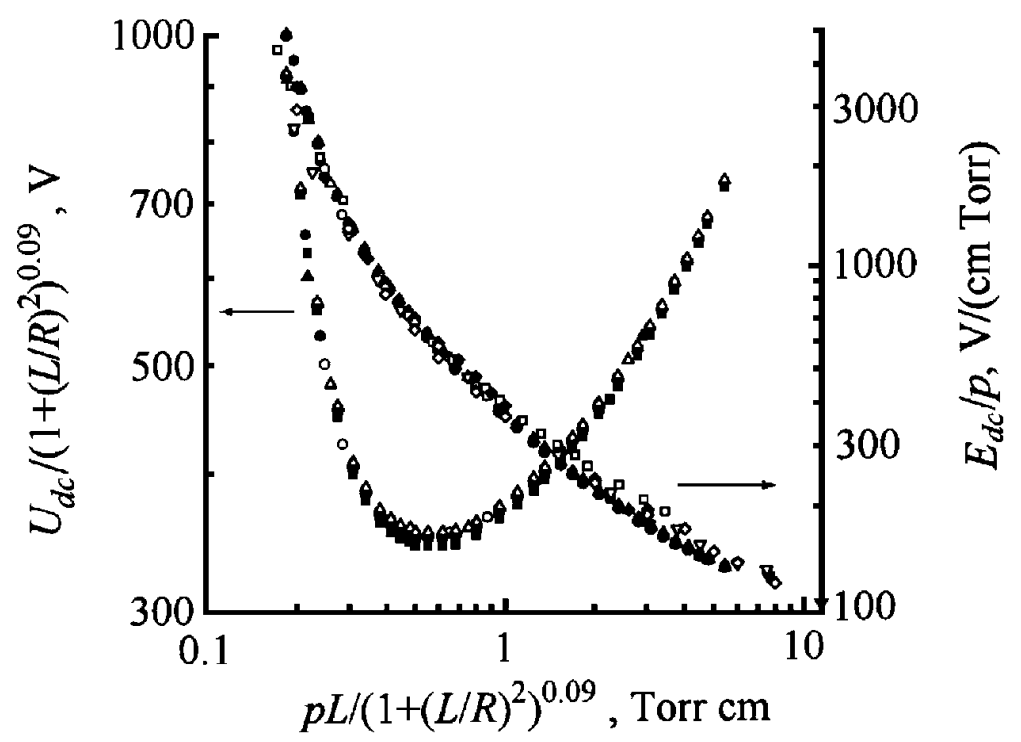

Figure 11. Dependences of $U_{d c}^{*}$ and $E_{d c} / p$ on $(p L)^{*}$ in air in the discharge tube with $R=3.15 \mathrm{~cm}$ and different interelectrode gaps:

$\bullet L=0.5 \mathrm{~cm}, \bigcirc L=1 \mathrm{~cm}, \boldsymbol{\Delta} L=2 \mathrm{~cm}, \square L=5 \mathrm{~cm}, \boldsymbol{\nabla} L=10 \mathrm{~cm}, \square$ measured data of [35], $\nabla$ measured data of [48], $\nabla$ measured data of [45], measured data of [49] and $\diamond$ measured data of [15].

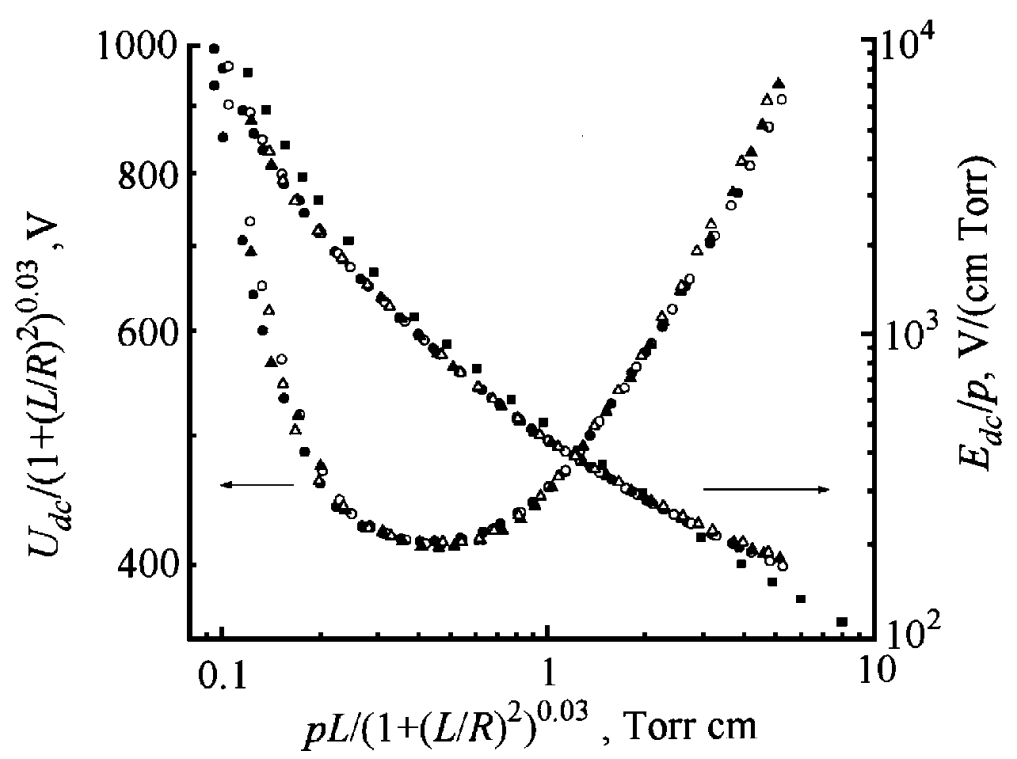

Figure 12. Dependences of $U_{d c}^{*}$ and $E_{d c} / p$ on $(p L)^{*}$ in oxygen in the discharge tube with $R=3.15 \mathrm{~cm}$ and different interelectrode gaps:

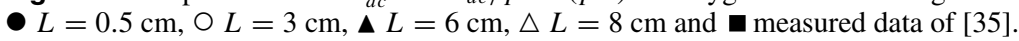

$(p L)_{\text {min }}^{*} \approx 0.6$ Torr $\mathrm{cm}$. From the relations (17) and (18) we obtain

$$
\begin{aligned}
(p L)_{\min } & =(p L)_{\min }^{*}\left[1+\left(\frac{L}{R}\right)^{2}\right]^{0.12} \\
U_{\text {min }} & =U_{\text {min }}^{*}\left[1+\left(\frac{L}{R}\right)^{2}\right]^{0.12} .
\end{aligned}
$$

For $L / R=1.59$ we have $U_{\text {min }} \approx 323 \mathrm{~V}$ and $(p L)_{\text {min }} \approx$ 0.7 Torr $\mathrm{cm}$. It follows from our measured data that $U_{\min } \approx$ $320 \mathrm{~V},(p L)_{\text {min }} \approx 0.73$ Torr $\mathrm{cm}$, i.e. the satisfactory agreement is observed between the measured coordinates of the $\mathrm{dc}$ breakdown curve minimum and the data predicted from the relations (17)-(20). To predict any other point of the dc breakdown curve $U_{1}^{*}$ and $(p L)_{1}^{*}$, we act in a similar manner.
In the general case, to predict a dc breakdown curve in a cylindrical discharge vessel with arbitrary geometric dimensions one should have the Paschen's curve (i.e. the dc breakdown curve measured in the vessel with $L / R \rightarrow 0$ with the cathode made out of a required material) and then with the help of the relations (17) and (18) determine the $U_{d c}$ and $p L$ values. If the initial dc breakdown curve was measured in the discharge vessel with $L_{0}$ and $R_{0}$, for which the condition $L_{0} / R_{0} \rightarrow 0$ is not valid, one should first construct the dependence $U_{d c}^{*}=$ $f\left((p L)^{*}\right)$ with the help of relations (17) and (18). Then, one should use this and the same relations (17) and (18) to calculate the sought breakdown curve in the discharge vessel with the given dimensions $L_{1}$ and $R_{1}$. It may be expressed more 
briefly with the help of the following relations:

$$
\begin{aligned}
& (p L)_{1}=p L_{0}\left[\frac{1+\left(L_{1} / R_{1}\right)^{2}}{1+\left(L_{0} / R_{0}\right)^{2}}\right]^{a} \\
& U_{d c 1}=U_{d c 0}\left[\frac{1+\left(L_{1} / R_{1}\right)^{2}}{1+\left(L_{0} / R_{0}\right)^{2}}\right]^{a}
\end{aligned}
$$

where the subscript 1 relates to the sought dc breakdown curve and the subscript 0 relates to the initial breakdown curve that was known.

In the expressions (17) and (18), the quantities $(p L)^{*}$ and $U_{d c}^{*}$ are the functions of the argument $(L / R)^{2}$, because the ratio of the interelectrode distance to the chamber radius enters equation (6) in the second power. However, the insulating wall of the discharge chamber collects the positive as well as the negative charges that are generated due to ionization and are lost on chamber walls due to diffusion. Therefore the losses of charged particles on the walls would be proportional to $(L / R)$, equivalent to the ratio of the wall area to that of the discharge cross section. In this case the quantities $(p L)^{*}$ and $U_{d c}^{*}$ may be rewritten as the functions of $(L / R)$ :

$$
\begin{aligned}
& (p L)^{*}=p L /\left[1+\frac{L}{R}\right]^{b} \\
& U_{d c}^{*}=U_{d c} /\left[1+\frac{L}{R}\right]^{b}
\end{aligned}
$$

where for oxygen, nitrogen, argon and air the values of $b$ are equal to $0.06,0.17,0.23$ and 0.16 , respectively. The expressions (17), (18), (23) and (24) are empirical and are obtained for the convenient processing of measured breakdown curves. Therefore one is free to choose between the different presentations.

Inside the burning dc discharge a negative surface charge $[5,9,50]$ is deposited on the tube walls. In a steady-state regime the electron and ion fluxes on the walls are equal. At the stage before the breakdown (Townsend regime), when the quasineutral plasma is not yet formed, the concentration of positive ions considerably exceeds that of the electrons [9], therefore the ion and electron fluxes to the walls are not equal. As was shown in [51], before the breakdown a distribution of charge may be formed on the internal surface of the tube due to tube conductivity. Consequently, a correct account of the effect of surface charge on the walls on the dc gas breakdown is associated with a complicated theoretical treatment, so we did not attempt to model this within the framework of this study. Some results in this important field may be found in [51-57].

Let us make a remark concerning the technique of measuring the breakdown curves. As a rule, one measures a breakdown curve of the glow discharge in two ways: (1) one fixes the gap $L$ and then varies the gas pressure $p$ and measures the breakdown voltage $U_{d c}$ or (2) one fixes the gas pressure and measures the breakdown voltage values for different gap values $L$. However, it follows from the results obtained in the present paper that the second method (with pressure fixed and gap width varying) is incorrect. The 'breakdown curve' obtained in this way will be a set of points each of which belongs to a certain genuine breakdown curve characterized by a fixed $L / R$ value. At small $L$ it is close to Paschen's, but with the gap values increasing it will shift to higher breakdown voltages. Therefore one should measure a dc breakdown curve by establishing a fixed interelectrode gap and varying the gas pressure.

\section{Conclusions}

This paper presents the results of the experimental and theoretical study of breaking down a low-pressure dc discharge in argon, nitrogen, air and oxygen for different interelectrode gaps, discharge tube radii and cathode materials. The ratio $\left(E_{d c} / p\right)_{\min }$ at the minimum of the breakdown curves is shown to be constant for any interelectrode gap $L$, discharge vessel radius $R$ and the ion-electron emission rate from the cathode surface $\gamma$. A modified dc breakdown law is obtained: $U_{d c}=$ $f(p L, L / R)$; i.e. the breakdown voltage $U_{d c}$ depends on the product of the gas pressure $p$, the gap width $L$ and on the ratio $L / R$. It is shown in experiment that the conventional Paschen's law, $U_{d c}=f(p L)$, is valid only for those discharge tubes, for which the electrode dimensions and interelectrode gaps are geometrically similar, and for short discharge tubes for which $L / R \rightarrow 0$. In general, Paschen's law is not valid. A method is presented which can predict the breakdown curve in a cylindrical vessel for any values of $L$ and $R$ from the measured data on a dc breakdown.

\section{References}

[1] Paschen F 1889 Annalen der Physik und Chemie 3769

[2] Townsend J S 1915 Electricity in Gases (Oxford: Clarendon)

[3] Druyvesteyn M J and Penning F M 1940 Rev. Mod. Phys. 12 87

[4] Meek J M and Craggs J D 1953 Electrical Breakdown of Gases (Oxford: Clarendon)

[5] Von Engel A 1955 Ionized Gases (Oxford: Clarendon)

[6] Llewellyn Jones M A 1956 Ionization growth and breakdown Handbuch der Physik vol 22, ed S Flugge (Berlin: Springer) $\mathrm{p} 1$

[7] Loeb L B 1956 Electrical breakdown of gases with steady or direct current impulse potentials Handbuch der Physik ed S Flugge (Berlin: Springer) p 445

[8] Brown S C 1959 Basic Data of Plasma Physics (New York: Wiley)

[9] Raizer Y P 1991 Gas Discharge Physics (Berlin: Springer)

[10] Lisovsky V A and Yegorenkov V D 1994 J. Phys. D: Appl. Phys. 272340

[11] Phelps A V and Petrovic Z Lj 1999 Plasma Sources Sci. Technol. 8 R21

[12] Sato M 1999 Bull. Yamagata Univ. 25119

[13] De La Rue W and Muller H W 1880 Phil. Trans. R. Soc. 171 65

[14] Townsend J S and McCallum S P 1928 Phil. Mag. 6857

[15] Fricke H 1933 Z. Phys. 86464

[16] McCallum S P and Klatzow L 1934 Phil. Mag. 17279

[17] Buttner H 1939 Z. Phys. 111750

[18] Heymann F G 1950 Proc. Phys. Soc. 6325

[19] Miller H C 1964 Physica 302059

[20] Jacques L and Bruynooghe W 1981 Proc. 15th Int. Conf. on Phenomena in Ionized Gases (Minsk) p 609

[21] Jacques L, Bruynooghe W, Boucique R and Wieme W 1986 J. Phys. D: Appl. Phys. 191731

[22] Yumoto M, Sakai T, Ebinuma Y, Fujiwara Y and Aihara M 1993 Proc. 8th Int. Symp. on High Voltage Engineering (Yokohama) p 409 
[23] Auday G, Guillot P, Galy J and Brunet H 1998 J. Appl. Phys. 835917

[24] Schofield J M S 1974 Proc. 3rd Int. Conf. on Gas Discharges (London) p 329

[25] Schofield J M S 1974 Proc. 4th Int. Conf. on Gas Discharges (Wales) p 397

[26] Slottow H H 1976 IEEE Trans. Electron. Devices 23760

[27] Schofield J M S 1980 PhD Thesis University of Surrey, UK

[28] Chen F F 1995 Phys. Plasmas 22164

[29] Boeuf J P, Punset C, Hirech A and Doyeux H 1997 J. Physique IV 7 C4 3

[30] Kolobov V I and Fiala A 1994 Phys. Rev. E 503018

[31] Ward A L 1962 J. Appl. Phys. 332789

[32] Schofer R 1938 Z. Phys. 11021

[33] Guseva L G 1970 Zh. Tekh. Fiz. 402253 (in Russian)

[34] Dikidgi A N and Klarfeld B N 1955 Zh. Tekh. Fiz. 251038 (in Russian)

[35] Schonhuber M J 1969 IEEE Trans. Power Apparatus Syst. 88 100

[36] Ehrenkranz F 1939 Phys. Rev. 55219

[37] Pace J D and Parker A B 1973 J. Phys. D: Appl. Phys. 61525

[38] Jacobs H and LaRocque A P 1947 J. Appl. Phys. 18199

[39] Jacobs H and LaRocque A P 1948 Phys. Rev. 74163

[40] Kucukarpaci H N and Lucas J 1981 J. Phys. D: Appl. Phys. 142001

[41] Puech V and Torchin L 1986 J. Phys. D: Appl. Phys. 192309

[42] Suzuki M, Taniguchi T and Tagashira H 1990 J. Phys. D: Appl. Phys. $\mathbf{2 3} 842$
[43] Lisovskiy V A and Yegorenkov V D 1998 J. Phys. D: Appl. Phys. 313349

[44] Phelps A V and Jelenkovic B M 1988 Phys. Rev. E 382975

[45] Dakin T W, Gerhold J, Krasucki Z, Luxa G, Oppermann G, Vigreux J, Wind G and Winkelnkemper H 1977 Proc. Int Conf. on Large High Voltage Electric Systems (Paris) p 1

[46] Held B, Soulem N, Peyrous R and Spyrou N 1996 Trans. IEE Japan A 116925

[47] Held B, Soulem N, Peyrous R and Spyrou N 1997 J. Physique III 72059

[48] Pim J A 1949 Proc. IEE 96117

[49] Ritz H 1937 Arch. Electrotech. 26219

[50] Behnke J F, Bindemann T, Deutsch H and Becker K 1997 Contrib. Plasma Phys. 37345

[51] Altrichter B and Arndt R 1974 Beitr. Plasmaphys. 14139

[52] Nedospasov A V and Novik A E 1961 Sov. Phys.-Tech. Phys. 51261

[53] Tunitskii L N and Cherkasov E M 1969 Zhurn. Tekh. Fiz. 39 2173 (in Russian)

[54] Grosu D, Bara St, Bruma C and Vartolomei C 1978 An. sti. Univ. Iasi, Sec. 1 b 2469

[55] Grosu D 1981 Proc. 15th Int. Conf. on Phenomena in Ionized Gases (Minsk) Contributed Paper $\mathrm{p} 601$

[56] Horstman R E and Oude Lansink F M 1988 J. Phys. D: Appl. Phys. 211130

[57] Xiang G S and Xiton O 1989 Proc. 19th Int. Conf. on Phenomena in Ionized Gases (Belgrade) Contributed Paper 4 p 896 